\title{
A PHASE TRANSITION MODEL AND TEMPORAL LOGIC SPECIFICATIONS FOR SMART ENERGY SYSTEMS - REVISITED
}

\author{
Byungkwon Park \\ Mohammed M. Olama \\ Computational Sciences and \\ Engineering Division \\ Oak Ridge National Laboratory \\ Oak Ridge, TN, 37831 USA \\ parkb@ornl.gov, olamahussemm@ornl.gov
}

\begin{abstract}
In this paper, we revisit the method of a phase transition model for representing hybrid systems and temporal logic specifications (TLSs) for specifying desired behaviors of systems, and discuss their usefulness for smart energy systems. On the one hand, the phase transition model incorporates the continuous model of the relay device action with a particular structural form that allows for the construction of a single, global differential-algebraic equation for hybrid systems (thus smoothed hybrid systems). On the other hand, the TLS allows sophisticated descriptions of control specifications addressing both magnitude and time simultaneously, which has recently been applied to the control strategy of several types of continuous and hybrid systems. We provide high-level descriptions of each of the two techniques and present simulation results in the context of smart energy systems.
\end{abstract}

Keywords: Discrete events, hybrid systems, smart energy systems, temporal logic specifications.

\section{INTRODUCTION}

Modeling and simulation $(\mathrm{M} \& S)$ provide a powerful tool for capturing the behaviors of complex cyberphysical systems including robotics, communication networks, and electric power networks. As the scale of such engineered systems expands, and the importance of the reliable operation of these systems for society increases, $M \& S$ plays a vital role in understanding the expected behavior and vulnerability of such complex systems due to small and large scale failures of individual elements. The work here is motivated largely by the electric power systems application (Bergen and Vittal 2000). The effort to make the power grid "smarter" through improved communication, control, and computation helps more efficiently manage electrical power, but such smart energy systems can also introduce a wider range of component failures (Kassakian and Schalensee 2011) and the need of more sophisticated control specifications.

The objective of this paper is to review and describe the two M\&S techniques, 1) a phase transition model (DeMarco 2001) and 2) temporal logic specifications (TLSs) (Raman et al. 2014), such that they both have the potential to address the aforementioned issues for smart energy systems. Notice that the vulnerability of smart energy systems is further increased due to new, more complex components that may be exposed to new mechanisms of outage with growing complexity and continuous evolution in power systems. In this aspect, developing an efficient tool to represent such discrete events interacting with continuous dynamics described 
by ordinary differential equations is crucial to understand the level of vulnerability and design appropriate control strategies. To this end, we first illustrate the phase transition model that allows for the construction of a single, global differential-algebraic equation (DAE) enabling the transitions between multiple state spaces without partitioning those for hybrid systems. From an analytic perspective, while the rigorous treatment of DAEs with discontinuous right-hand sides is interesting and a challenging issue (DeCarlo et al. 2000), it has connections to the ideas presented here and is beyond the scope of this paper.

The TLS was originally developed to provide a systematic mathematical formulation for specifying and monitoring the expected behavior of physical systems including temporal constraints. Then, this technique has recently been applied to the control strategy of continuous and hybrid systems including frequency control in microgrids (Zhang et al. 2018). The existing works for designing control strategies incorporating TLSs can be characterized into two categories: one is to represent the system as a transition system and transform the controller synthesis problem into a series of constrained reachability problems (Wolff, Topcu, and Murray 2013), (Coogan et al. 2016), and the other is to represent the controller synthesis as a single optimal control problem and impose TLSs as optimization constraints (Saha and Julius 2016). The second category (optimization-based approach) is mainly used in continuous-time temporal logics, and is our focus for the TLS in this paper.

The key novelty of the phase transition model and TLSs is the machanism to enable smooth (and rapid) transitions between multiple state spaces and the incorporation of more sophisticated control specifications for smart energy systems, respectively. This paper revisits these two techniques with high level descriptions and provides simulation results in the context of smart energy systems. We discuss the contribution of each technique on smart grid monitoring with a high concentration of induction motors and frequency control addressing dwell time constraints. The balance of the paper is organized as follows. Section II reviews the phase transition model. Section III introduces preliminaries on the TLS and the problem statement for the optimal control scheduling problem using model-predictive control (MPC) with the TLS. Numerical case studies for each technique are summarized in Section V. The conclusion is presented in Section VI.

\section{A PHASE TRANSITION MODEL}

This section describes the phase transition model based on the work in (DeMarco 2001). In the scenario of interest, it restricts its attention to the systems that the transition (such as failure) of individual elements would be governed by threshold logic, and the transition of an element would induce a discontinuous change in the structure of the systems. The key concept of the phase transition model is based on the fact that the threshold failure mechanisms can be associated with energy-storing elements and that the threshold itself may be expressed in terms of energy stored in the element. That is, it seeks to capture the transient dynamic response following a specified initial disturbance and subsequent discrete events that result from the excess of operating thresholds for individual elements along the state trajectory.

With these concepts, the objective of the phase transition technique is to develop a model in which a single DAE can treat the interaction of multiple discrete events. To facilitate this goal in a fixed state space structure, it smooths the discontinuity of a discrete event.

\subsection{Smooth Function}

To begin with, the ideal binary variable to describe element in-service and element out-of-service is treated as a continuously varying state in a smooth differential equation. The approach is to introduce an indicator variable and a smooth function, which allows for the construction of the state equation for the indicator variable from a gradient of a smooth function. This yields a set of continuous ordinary differential equations such that it exhibits strongly "bistable" behavior, maintaining the value of the indicator variable in a 


\section{Park and Olama}

neighborhood near 1 when the element is operating normally, and forcing its value to a neighborhood near 0 during the discrete event. Notice that this smoothed model is constructed in a way that the state transition occurs (when triggered) much more rapidly than the dynamic evolution of states between discrete events.

Consider the continuous variable $\lambda_{k}$, which is the indicator variable associated with each element $k$. Our goal is to use this indicator variable to represent the state transition. That is, when the $k$ th element is in service and operates normally, $\lambda_{k}=1$. When the energy stored in the element $k$ exceeds its specified limit, the relay acts and transits its state. In this case, the variable $\lambda_{k}$ moves from 1 to 0 rapidly. Notice that the value of $\lambda_{k}$ changes continuously during very short time interval, acting as a binary variable. Thus, we call this variable as "bistable variable." To allow such smooth, but very fast transition, we introduce the switching function to smooth the transition between 1 and 0 of variable $\lambda_{k}$ for element $k$. The switching function vector $\theta(\lambda)$ can be defined as

$$
\theta(\lambda)=\left[\theta_{1}\left(\lambda_{1}\right), \cdots, \theta_{k}\left(\lambda_{k}\right)\right],
$$

where $\lambda \in \mathbb{R}^{K}, K$ representing the number of elements. In particular, switching functions for $\lambda$ can be defined as

$$
\theta_{k}\left(\lambda_{k}\right) \equiv 2\left[-e^{-20 \lambda_{k}}+e^{-200 \lambda_{k}}+e^{20\left(\lambda_{k}-1\right)}-e^{200\left(\lambda_{k}-1\right)}-0.2\right], \quad k \in K .
$$

This function is displayed in Fig. 1 , which shows when $\lambda \approx 1 \rightarrow \theta(\lambda) \approx 1$, and $\lambda \approx 0 \rightarrow \theta(\lambda) \approx 0$. Notice that this seemingly arbitrary function is carefully designed and constructed to impose a dynamic structure that yields two stable equilibria for each bistable variable. By construction (which will be discussed below), these equilibria will be placed in a neighborhood of 1 and in a neighborhood of 0 . In addition, this construction allows that 1) the threshold value that initiates the state transition can be parametrized in the model by appropriate scaling of $\theta(\lambda), 2$ ) the slopes in the vicinity of 1 and 0 can be modified with suitable adjustment of the coefficients of the exponential terms, forcing equilibrium values to lie as close to 0 or 1 as desired, and 3) a single, global Lyapunov function can be employed and facilitated for a stability analysis (although the details of this analysis are not covered in this paper). Readers may investigate other alternatives with the consideration of the above characteristics for this switching function to explore its potential.

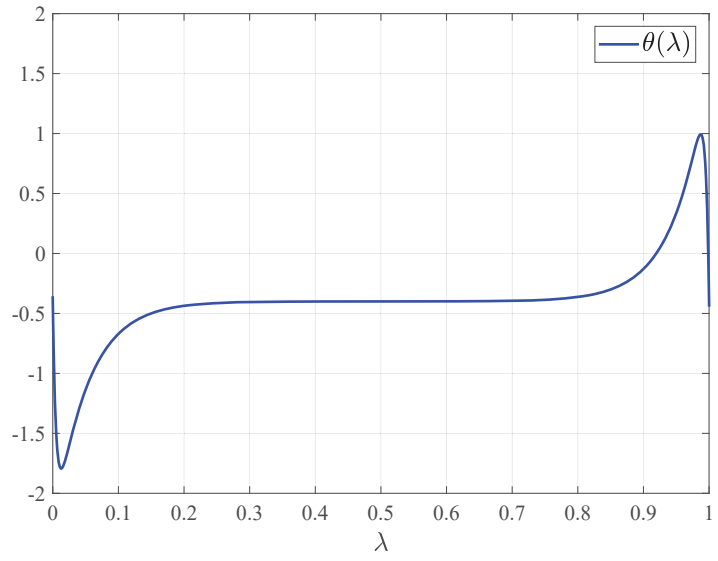

Figure 1: Illustration of $\theta(\lambda)$.

The integral of this function is also important to understand the behavior of this model. The integral of a component $\theta_{k}\left(\lambda_{k}\right)$ with respect to $\lambda_{k}$ is denoted $\Theta_{k}\left(\lambda_{k}\right)$, and is defined as:

$$
\Theta_{k}\left(\lambda_{k}\right) \equiv \int_{1}^{\lambda_{k}}-\theta\left(\eta_{k}\right) d \eta_{k}, \quad k \in K
$$




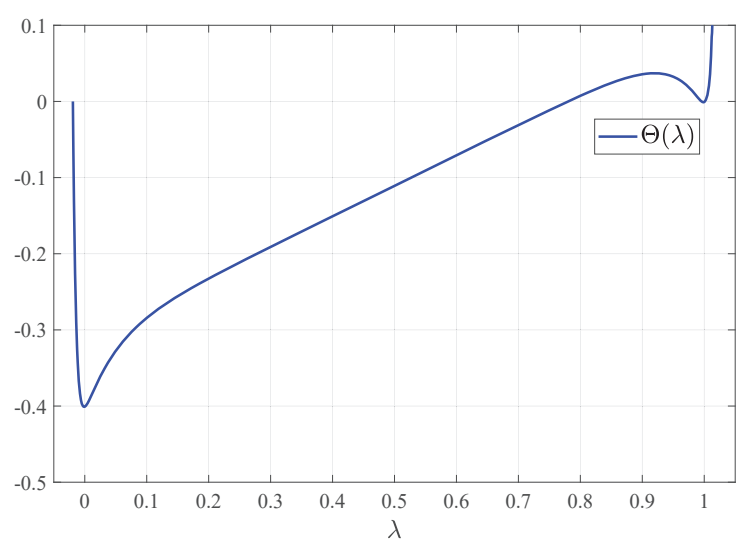

Figure 2: Illustration of $\Theta(\lambda)$.

This function is displayed in Fig. 2. Notice that the -0.4 offset comes from $\theta(\lambda)$, and this represents a net drop in energy of approximately -0.4 due to a transition of a element from in-service state to out-ofservice state. Importantly, the graph of $\Theta(\lambda)$ illustrates two equilibria points in the vicinity of 1 and 0 with a potential barrier that blocks the transition from 1 to 0 and vice versa. The height of the potential plays an important role; one should overcome the height of the potential to escape the local minimum and move to another local minimum. This value is set by the failure threshold, not by the choice of the offset term in the definition of $\theta(\lambda)$.

\subsection{Threshold Failure Model}

As discussed before, this section describes a threshold failure model, which requires that one can express the element failure threshold as a limiting value on the stored energy in the element. To illustrate, consider the example in the perspective of circuit analysis, such that the magnitude of a branch current $i$ exceeding a specified threshold would trigger the element failure. In a power system context, transmission lines will appear as inductive elements with series inductance $L$. Then, given an inductor current magnitude as a threshold, one can derive the energy stored in a lossless inductive branch as $\frac{1}{2} L i^{2}$.

With this energy storage term, the goal is to relate the relay action of an overloaded transmission line to the stored energy quantity. Suppose that the relay action opens a circuit breaker on a branch, resulting in the branch having infinite inductance. This indicates that the inverse of the inductance $L^{-1}$ goes to zero. Then, we associate the bistable variable $\lambda$ with the inverse of the inductance $L^{-1}$ to represent the relay action of a branch. Then, the energy stored in the inductor with the bistable variable $\lambda$ can be represented as:

$$
W=\frac{1}{2} \lambda L^{-1} I^{2}
$$

where $I=L i$, which can be interpreted as the magnetic flux. Notice that when $\lambda=1$, the branch is in service and the energy stored in the branch is equal to $\frac{1}{2} L^{-1} I^{2}$, and when $\lambda=0$, the branch is out of service and the energy stored in the branch is equal to 0 .

\subsection{Differential Equations for Smooth Transition}

This section combines the aforementioned two concepts, the smooth function and the threshold failure model, to derive different equations for smooth transitions (the phase transition model). To this end, suppose the derivative of $\lambda$ with respect to time is composed of the energy-like form minus $\theta(\lambda)$. Its general form 
(ordinary differential equation) can be represented as:

$$
\dot{\lambda_{k}}=-D_{\lambda, k}^{-1}\left(W_{k}(x)-R_{\lambda, k} \theta_{k}(\lambda)\right), \quad k \in K .
$$

where $W_{k}(x)>0$ represents the energy-like form for the element $k ; R_{\lambda, k}>0$ represents a pre-set threshold for $W_{k}(x)>0$; and $D_{\lambda, k}$ is a small positive time constant. Here $x$ can be any state variable, e.g., bus voltage magnitude and angle, line current or frequency deviation, etc. Notice that (4) expresses the relationship between the "energy" stored in the element $k$ and the bistable variable $\lambda_{k}$.

To understand the behavior of (4), at a normal operating point, assume that $\lambda_{k}$ is initiated near 1 , so that the initial $\lambda_{k}$ stays the steeply sloped region near 1 . In this scenario, $\lambda_{k}$ stays near 1 (thus, $\left.\theta_{k}\left(\lambda_{k}\right) \approx 1\right)$, as long as $R_{\lambda, k} \theta_{k}(\lambda)>W_{k}(x)$. If $W_{k}(x)$ exceeds the threshold $R_{\lambda, k}$ due to some event in the system, $\dot{\lambda_{k}}$ would become negative, causing $\lambda_{k}$ to decrease from 1 by turning the term $W_{k}(x)-R_{\lambda, k} \theta_{k}(\lambda)$ positive. The nature of $\theta_{k}\left(\lambda_{k}\right)$ and a nonnegative value of $W(x)$ ensure that $\dot{\lambda}_{k}$ become strongly negative once it drops below 1 , and $\lambda$ further decreases quickly until it is "captured" in a new equilibrium in the vicinity of $\lambda=0$.

Notice that $W(x)$ can have a similar form in (3) to represent the energy-like form, $R_{\lambda, k}$ is a constant value that one should define depending on the dynamic model considered, and any dynamic model described by DAEs can be augmented with (4) to associate bistable variables with each element that is subject to the possible state transition.

\section{TEMPORAL LOGIC SPECIFICATIONS}

This section briefly reviews TLSs based on the work in (Raman et al. 2014) and (Raman and Donze. 2016) that can be embedded in MPC. This technique may be particularly valuable for smart energy systems. Notice that smart energy systems are increasingly utilizing distributed energy resources (DERs) to more efficiently and reliably support grid services (Sun et al. 2019). In addition, it has been discussed that the microgrid (part of smart energy systems) has a potential for powering local locations and making the power grid more resilient against disturbances (Allen et al. 2016).

However, the satisfaction of certain performance specifications with various DERs having different response times and duration times is a challenging task. For example, such specifications include "after a fault is cleared, the system frequency should be restored back to $60 \mathrm{~Hz} \pm 0.2 \mathrm{~Hz}$ within $2 s^{\prime \prime}$ (Xu, Julius, and Chow 2019). This logic would become more important for the proper operation of microgrids in which most protection relays are designed and required to consider the status of states and their dwell times simultaneously. This argument can also be applied to voltage control in smart energy systems. In this scenario, the TLS can provide a powerful tool to satisfy various control guidelines including temporal logics.

For the description of the TLS in subsequent sections, we consider discrete-time continuous systems, described as:

$$
x_{t+1}=f\left(x_{t}, u_{t}\right),
$$

where $t=0,1, \ldots$ are time indices, $x$ represents the continuous and binary/logical states, and $u$ is the control inputs. A run $\mathbf{X}=x_{0} x_{1} x_{2} \ldots$ is an infinite sequence of its state, and for each $t=0,1, \ldots$, there exists a control input $u_{t}$ satisfying $x_{t+1}=f\left(x_{t}, u_{t}\right)$.

\subsection{Preliminaries on the TLS}

Let $\varphi$ be an atomic proposition that maps the system state variables to the Boolean domain $\mathbb{B}=$ $\{$ True, False $\}$. The syntax of a TLS describes the structure of the syntactic formula for logic, and the semantic of a TLS describes the meaning assigned to the formulas. The syntax of a TLS is defined recursively 
as follows:

$$
\varphi:=\gamma|\neg \gamma| \varphi \wedge \psi|\varphi \vee \psi| \Rightarrow \mid \Leftrightarrow,
$$

where $\gamma$ is a predicate whose truth value is determined by the sign of a function of an underlying signal $x$, i.e., $\gamma \equiv \gamma(x)>0 ; \psi$ is a TLS formula; and $\neg, \wedge, \vee, \Rightarrow, \Leftrightarrow$ are negation, conjunction, disjunction, implication, and equivalence logical operators, respectively. The standard temporal operators used in TLSs are $\diamond_{\left[t_{1}, t_{2}\right]}$ (eventually) and $\square_{\left[t_{1}, t_{2}\right]}$ (always) such that $\square_{\left[t_{1}, t_{2}\right]} \varphi$ means $\varphi$ holds at every time step between $t_{1}$ and $t_{2}$, and $\diamond_{\left[t_{1}, t_{2}\right]} \varphi$ means $\varphi$ holds at some time step between $t_{1}$ and $t_{2}$.

We denote $x \mid=\varphi$ if a signal $x$ satisfies $\varphi$. For example, $x=\square_{\left[t_{1}, t_{2}\right]} \varphi$ if the formula defined by $\varphi$ holds at every time step between $t_{1}$ and $t_{2}$. The validity of a formula $\varphi$ with respect to signal $x$ at time $t$ is defined as follows:

$$
\begin{array}{lll}
(x, t) \models \gamma & \Leftrightarrow \gamma\left(x_{t}\right)>0 . \\
(x, t) \models \neg \gamma & \Leftrightarrow \neg((x, t) \models \gamma) . \\
(x, t) \models \varphi \wedge \psi & \Leftrightarrow \quad(x, t) \models \varphi \wedge(x, t) \models \psi . \\
(x, t) \models \varphi \vee \psi & \Leftrightarrow \quad(x, t) \models \varphi \vee(x, t) \models \psi . \\
(x, t) \models \square_{\left[t_{1}, t_{2}\right]} \varphi & \Leftrightarrow \quad \forall t^{\prime} \in\left[t+t_{1}, t+t_{2}\right],\left(x, t^{\prime}\right) \models \varphi . \\
(x, t) \models \diamond_{\left[t_{1}, t_{2}\right]} \varphi & \Leftrightarrow \exists t^{\prime} \in\left[t+t_{1}, t+t_{2}\right],\left(x, t^{\prime}\right) \models \varphi .
\end{array}
$$

\subsection{Algorithmic Formulation for the TLS}

For a formula $\varphi$, we introduce a variable $z_{t}^{\varphi}$ that has an associated set of mixed integer linear programming (MILP) constraints such that $z_{t}^{\varphi}=1$ if and only if $\varphi$ holds at position $t$.

\subsubsection{Predicates}

Notice that constraints on system state variables represent the predicates. For each predicate $\gamma$, binary variables $z_{t}^{\gamma}$ are introduced for time $t=0,1, \ldots, K$. Then, the condition that $z_{t}^{\gamma}=1$ if and only if $\gamma\left(x_{t}\right)>0$ is enforced by following two constraints:

$$
\begin{aligned}
\gamma\left(x_{t}\right) & \leq M_{t} z_{t}^{\gamma}-\varepsilon_{t}, \\
-\gamma\left(x_{t}\right) & \leq M_{t}\left(1-z_{t}^{\gamma}\right)-\varepsilon_{t},
\end{aligned}
$$

where $M_{t}$ are sufficiently large positive numbers, and $\varepsilon_{t}$ are sufficiently small positive numbers to bound $\gamma\left(x_{t}\right)$ away from 0 . Note that (6) ensures that $z_{t}^{\gamma}=1$ if and only if $\gamma\left(x_{t}\right)>0$. This restricts TLS formulas to linear predicates, but admits arbitrary TLS formulas over such predicates.

\subsubsection{Boolean Operations}

As illustrated previously, each predicate $\gamma$ has an associated binary variable $z_{t}^{\gamma}$. Similarly, each $\varphi$ in a Boolean operation has a corresponding variable $z_{t}^{\varphi} \in[0,1]$, which is 1 if $\varphi$ holds at $t$ and 0 if not. Here, it also defines Boolean operations on these variables. 
Logical operations on variables $z_{t}^{\psi} \in[0,1]$ are defined as follows:

$$
\begin{array}{rr}
\text { Negation: } z_{t}^{\psi}=\neg z_{t}^{\varphi} & \Rightarrow z_{t}^{\psi}=1-z_{t}^{\varphi} . \\
\text { Conjunction: } z_{t}^{\psi}=\wedge_{i=1}^{j} z_{t_{i}}^{\varphi_{i}} & \Rightarrow z_{t}^{\psi} \leq z_{t_{i}}^{\varphi_{i}}, i=1, \ldots, j . \\
& z_{t}^{\psi} \geq 1-j+\Sigma_{i=1}^{j} z_{t_{i}}^{\varphi_{i}} . \\
\text { Disjunction: } z_{t}^{\psi}=\vee_{i=1}^{j} z_{t_{i}}^{\varphi_{i}} & \Rightarrow z_{t}^{\psi} \geq z_{t_{i}}^{\varphi_{i}}, i=1, \ldots, j . \\
& z_{t}^{\psi} \leq \Sigma_{i=1}^{j} z_{t_{i}}^{\varphi_{i}} .
\end{array}
$$

Given a formula $\psi$ with a Boolean operation, it introduces new continuous variables $z_{t}^{\psi} \in[0,1]$. Then, (7) ensures that for each Boolean operation, $z_{t}^{\psi}=1$ if $\psi$ holds at time $t$ and $z_{t}^{\psi}=0$ otherwise.

\subsubsection{Temporal Constraints}

Formulations for the $\square$ and $\diamond$ temporal operators are as follows:

$$
\begin{aligned}
& \text { Always: } \psi=\square_{\left[t_{1}, t_{2}\right]} \varphi \text {, } \\
& \text { let } a_{t}^{K}=\min \left(t+t_{1}, K\right) \text { and } b_{t}^{K}=\min \left(t+t_{2}, K\right) \text {. } \\
& \text { define } z_{t}^{\psi}=\wedge_{i=a_{t}^{K}}^{b_{t}^{K}} z_{i}^{\varphi} \text {. } \\
& \text { Eventually: } \psi=\diamond_{\left[t_{1}, t_{2}\right]} \varphi \text {, } \\
& \text { define } z_{t}^{\psi}=\vee_{i=a_{t}^{K}}^{b_{t}^{K}} z_{i}^{\varphi} .
\end{aligned}
$$

As an example, suppose one wishes to satisfy $\square\left(x_{t}<2\right)$ indicating $x<2$ always. The resulting predicates are $\gamma\left(x_{t}\right)=-\left(x_{t}-2\right)$ for time $t=0, \ldots, K$. Then, for each predicate (i.e., each time), the binary variables $z_{t}^{\gamma}$ are introduced with (6). For "always," additional constraints (7b), (7d) with the binary variable $z_{t}^{\text {alw }}$ should be added. When an interval is considered, follows (8a) with appropriate $a, b$ values. These constraints enforce that with $z_{t}^{\text {alw }}=1$ the formula $\gamma$ is satisfied at every time step on the interval $[a, b]$ relative to time step $t$. Its graphical illustration for "always" is depicted in Fig. 3. Similarly, for "eventually" with (7d), (7e), (8b), these constraint enforce that with $z_{t}^{\mathrm{ev}}=1$ the formula $\gamma$ is satisfied at some time step (since at least one of $z_{[a, b]}^{\gamma}$ is 1$)$ on the interval $[a, b]$ relative to time step $t$.

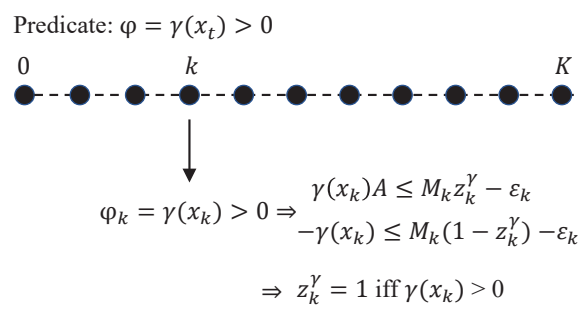

(a) Predicate

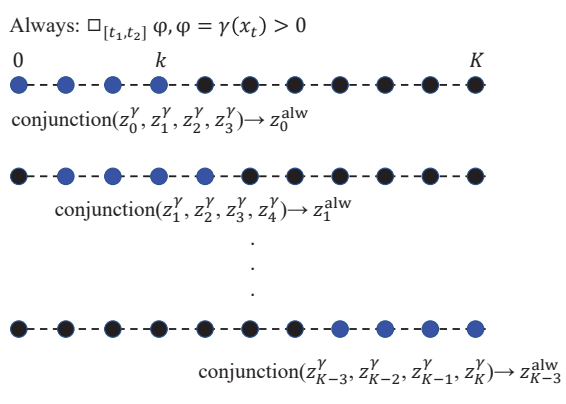

(b) Temporal operator with always

Figure 3: Illustration of the TLS.

The union of the TLSs and system constraints yields an MILP problem, enabling checking the feasibility of these constraint sets and finding a solution using an MILP solver. Given an objective function, this approach also provides an optimal trajectory that satisfies the TLSs. 


\subsection{Control Design}

Now, we formally state the TLS control synthesis problem and its MPC formulation. Suppose that the state space model is given (can also be obtained as a linearization of (5)) with a sample time of $t_{s}$ and expressed compactly as follows:

$$
\begin{aligned}
x_{t+1} & =A x_{t}+B u_{t}+E w_{t}, \\
y_{t} & =C x_{t}+D u_{t}+F w_{t},
\end{aligned}
$$

where $t_{0}=0$, and $x, u, w, y$ represent the system state, control input, external input, and system output, respectively.

Here we denote a control input sequence $\mathbf{u}=u_{0} u_{1}, \ldots, u_{K-1}$. Then, given a TLS formula $\varphi$ and a horizon $0<H<K$, for all $0 \leq t \leq K-H$, the optimal control problem computes an optimal $u_{t}^{*}$ as the first element of the sequence $\mathbf{u}_{t}^{H *}=u_{t}^{H *} u_{t+1}^{H *}, \ldots, u_{t+H-1}^{H *}$ satisfying

$$
u_{t}^{H *}=\operatorname{argmin} C\left(x_{t}, \mathbf{u}_{t}^{H}\right)=\sum_{k=0}^{H-1}\left|u_{t}\right|,
$$

subject to

$$
\begin{aligned}
x_{t+1} & =A x_{t}+B u_{t}+E w_{t}, \\
y_{t} & =C x_{t}+D u_{t}+F w_{t}, \\
\left|u_{t}\right| & \leq u^{\max }, \\
x_{t} & =\varphi,
\end{aligned}
$$

where the objective is to minimize the control efforts; (9d) ensures that the control input should not exceed a certain limit at any time; and (9e) states the TLS constraints. The parameters of this MILP are the initial state $x_{0}$ and external input vector $w$. With these, a solver can compute an optimal solution $\mathbf{u}^{*}$ for horizon $H$ which is passed and used by the state-space model. In MPC, only the first optimal input is used by the model for one time step, and the process is reiterated. Fig. 4 illustrates the corresponding closed-loop control scenario.

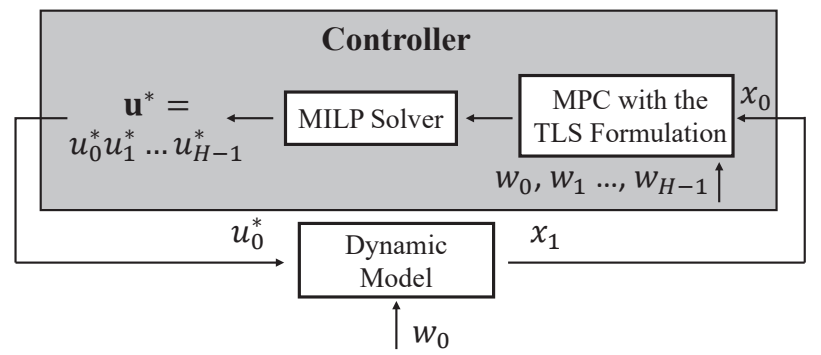

Figure 4: Closed-loop control synthesis with MPC.

\section{SIMULATION RESULTS}

This section investigates the two illustrated techniques on two examples of smart energy systems. For the phase transition model, we consider the fault-induced delayed voltage recovery (FIDVR) phenomenon using the IEEE 57 bus test system. For the TLS, we consider frequency control in a microgrid using a simplified microgrid test case consisting of one diesel generator (DG) and two wind turbine generators (WTGs). 


\subsection{The Phase Transition Model for Simulation of the FIDVR phenomenon}

In a distribution network, FIDVR issues resulting from a high concentration of induction motor loads have been very common and become concerning due to their potential threats to the transmission network (NERC 2009). Over-simplifying slightly, the FIDVR is mainly caused by stalled induction motors due to a faultinduced, temporary depressed voltage. These stalled induction motors draw large amount of reactive power, further depressing local voltages and delaying the voltage recovery after the fault is cleared.

Developing an appropriate time domain simulation model is an important task for studying FIDVR phenomenon, and one approach is to consider multiple static performance models and incorporate the transition between them. Based on the work (Zheng and DeMarco 2016), we seek to duplicate the FIDVR event using the phase transition model. In particular, we focus on two states: 1) $\lambda=0$ for motor operating at normal and 2) $\lambda=1$ for motor stalling triggered by a fault in the system. Notice that a large amount of reactive power demand $\left(Q^{\text {stall }}\right)$ occurs when the motor stalls. The general dynamic model for the logic of this FIDVR event can be represented as:

$$
\dot{x}= \begin{cases}f(x), & \text { for normal operating state. } \\ f(x)+Q^{\text {stall }}, & \text { for stalling state. }\end{cases}
$$

where $x$ represents the state variable of the network, and the function $f$ represents the network dynamic. Here the index for buses and network elements is dropped to minimize the notational clutter. That is, the term $Q^{\text {stall }}$ only appears at load buses having induction motors.

To represent the hybrid system (10) as a single DAE, we employ the phase transition model with the indicator variable $\lambda$, described as:

$$
\begin{aligned}
& \dot{x}=f(x)+Q^{\text {stall }} \lambda, \\
& \dot{\lambda}=-D_{\lambda}^{-1}\left(\frac{1}{2} J_{1} V^{2}-J_{\lambda}-R_{\lambda} \theta(\lambda)\right),
\end{aligned}
$$

where $V$ is the voltage magnitude at the load bus having inductor motors; $J_{\lambda}$ and $R_{\lambda}$ are all user-specified threshold constants; and $\theta(\lambda)$ is the switching function defined in (1). Notice that $\frac{1}{2} J_{1} V^{2}$ is the energy-like form in this example.

For the simulation, we include one induction motor in the system and consider the following scenario: 1) when $t=0$, the system is operating at steady state with the motor operating normally with $\lambda=0,2$ ) when $t=1 \mathrm{~s}$, a resistive bus-to-ground fault is applied for 0.2 seconds, resulting in the voltage drop at the load bus having this induction motor. Due to this voltage drop, the induction motor stalls with $\lambda=1$ and draws a large amount of reactive power.

The voltage drops at the load bus having the induction motor with and without FIDVR are depicted in Fig. 5. Notice that after the fault, the voltage starts to drop, and it gradually recovers and converges to the normal operating point without the FIDVR, shown in Fig. 5a, whereas the FIDVR causes the delay of voltage recovery, shown in Fig. 5b. The understanding of this FIDVR event with (11) is that:

- $\quad$ Before the fault, the term $\left(\frac{1}{2} J_{1} V^{2}-J_{\lambda}-R_{\lambda} \theta(\lambda)\right)$ is positive, so that $\lambda$ stays near 0 .

- After the fault, the voltage $V$ starts to decrease, turning the term $\left(\frac{1}{2} J_{1} V^{2}-J_{\lambda}-R_{\lambda} \theta(\lambda)\right)$ negative, causing $\lambda$ to increase from 0 to 1 .

- Then, with $\lambda=1, Q^{\text {stall }}$ becomes active in (11), acting as a considerable reactive power consumption from the stalled motor. Due to this, the recovery of the depressed load bus voltage faces the delay.

The corresponding smooth transition for the bistable variable $\lambda$ is shown in Fig. 6 . 


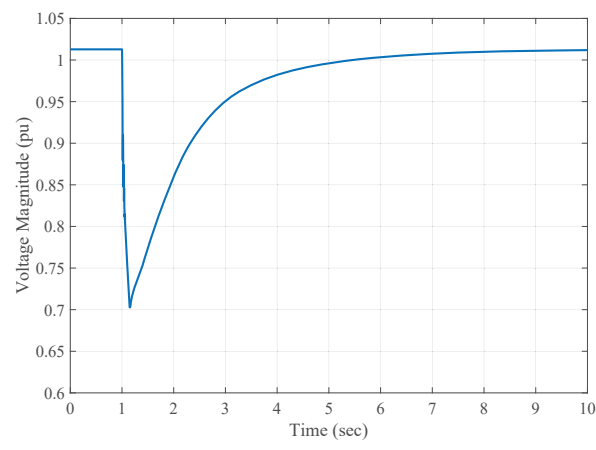

(a) Without the FIDVR.

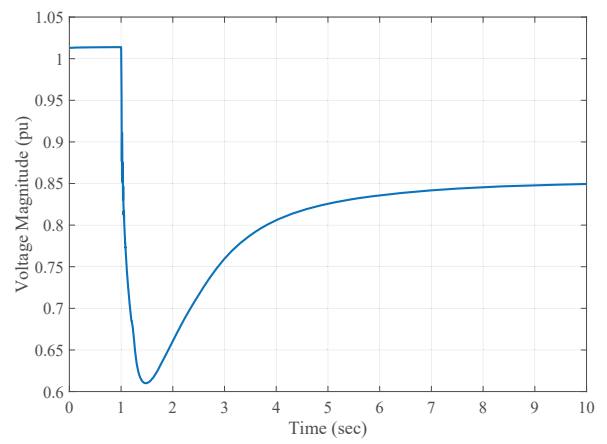

(b) With the FIDVR.

Figure 5: Voltage plots at the load bus having the induction motor.

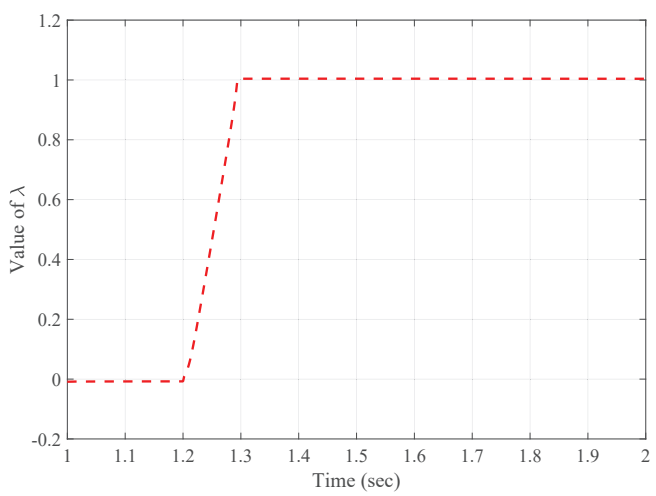

Figure 6: Bistable variable $\lambda$.

\subsection{Temporal Logic Specifications for Frequency Control in Microgrids}

Next, we consider the TLS for frequency control in a microgrid. For the verification, we employ a simple test network that one DG and two WTGs are connected to a single bus based on the work in (Zhang et al. 2018). In the context of frequency control, our focus is the speed of the DG, and we require the frequency deviation $\Delta f$ to be limited within $0.5 \mathrm{~Hz}$. In addition, we are interested in the logic that "whenever the frequency $\Delta f$ is larger than 0.45 , then it should become less than 0.45 in less than 1 seconds for always." This logic can be represented as

$$
\varphi=\square\left[(|\Delta f(t)| \geq 0.45) \Rightarrow\left(\diamond_{[0,1]} \square(|\Delta f(t)| \leq 0.45)\right)\right] .
$$

Suppose that the state space matrices (A, B, C, D, E, F) for the microgrid network dynamics are given. Then, the logic (12) can be inserted into (9e) to achieve the specified control specification. The resulting simulations are depicted in Fig. 7, which demonstrates that the resulting frequency response utilizing the TLS strictly satisfies the specified control specification with minimum control efforts required.

\section{CONCLUSION}

This paper revisited two M\&S techniques, the phase transition model and temporal logic specifications, and discussed their values in the context of smart energy systems. The phase transition model provides a special structure of dynamic system model that admits a very tractable inclusion of discrete events. This technique 


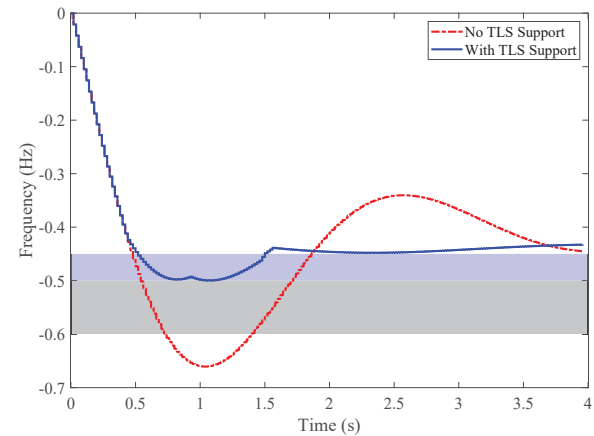

(a) Frequency of DG.

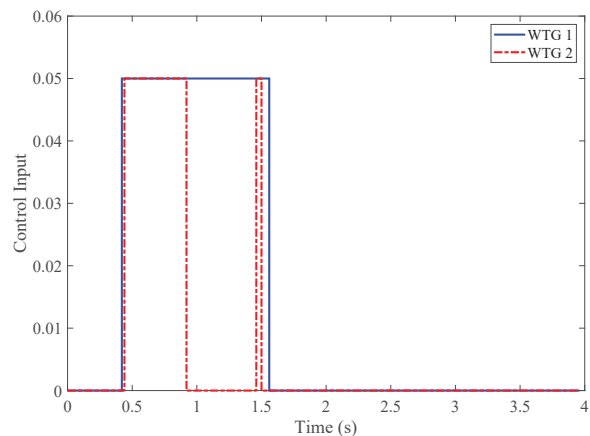

(b) Scheduled control signals for WTGs.

Figure 7: Frequency and control input signals with and without the TLS.

incorporates switching functions to mimic threshold relay actions and allows for the construction of a single, global DAE for such hybrid systems. Using a power system network as a test case, this work described how the smooth transition can be constructed.

In addition, this article reviewed the TLS to achieve control specification imposing constraints on not only the magnitude, but also the time simultaneously. We discussed the syntax of the TLS to describe the structure of the syntactic formula for logic, and detailed the corresponding mathematical formulations for the TLS, which can be integrated with MPC. Using the example of the microgrid, this article demonstrated its sophisticated control capability.

For future work, the phase transition model can be further utilized to consider more challenging and interesting hybrid systems such as cascading network failures. Its versatility to facilitate the transition between multiple discrete events could provide a suitable tool for predicting and preventing cascading failures in large-scale networks. In the context of the TLS, it would be interesting to design a tighter polytope (description) of TLS constraints to improve the computational performance of its optimization problem. Moreover, we want to consider a wider variety of smart energy systems such as smart building management systems requiring more sophisticated control performance.

\section{ACKNOWLEDGMENTS}

This manuscript has been authored by UT-Battelle, LLC under Contract No. DE-AC05-00OR22725 with the U.S. Department of Energy. The United States Government retains and the publisher, by accepting the article for publication, acknowledges that the United States Government retains a non-exclusive, paid-up, irrevocable, world-wide license to publish or reproduce the published form of this manuscript, or allow others to do so, for United States Government purposes. The Department of Energy will provide public access to these results of federally sponsored research in accordance with the DOE Public Access Plan (http://energy.gov/downloads/doe-public-access-plan).

\section{REFERENCES}

Allen, R., D. Brutkoski, D. Farnsworth, and P. H. Larsen. 2016. "Sustainable energy solutions for rural alaska". Technical Report LBNL-1005097, Lawrence Berkeley Nat. Lab.

Bergen, A. R., and V. Vittal. 2000. Power System Analysis. 2nd ed. Prentice Hall.

Coogan, S., E. A. Gol, M. Arcak, and C. Belta. 2016. "Traffic network control from temporal logic specifications”. IEEE Trans. Control Netw. Syst. vol. 3 (2), pp. 162-172. 
DeCarlo, R. A., M. S. Branicky, S. Pettersson, and B. Lennartson. 2000. "Perspectives and results on the stability and stabilizability of hybrid systems". Proc. IEEE vol. 88, pp. 1069-1082.

DeMarco, C. L. 2001. "A Phase Transition Model for Cascading Network Failure". IEEE Control Systems Magazine vol. 21 (6), pp. 40-51.

Kassakian, J., and R. Schalensee. 2011. "The future of the electric grid”. Technical report, Massachusetts Institute of Technology, Cambrdige, MA, USA.

NERC 2009. "A Technical Reference Paper Fault-Induced Delayed Voltage Recovery”. Technical report.

Raman, V., and A. Donze. 2016. "BluSTL: A Model Predictive Control Toolbox with Signal Temporal Logic Constraints". https://github.com/BluSTL/BluSTL.

Raman, V., A. Donze, M. Maasoumy, R. M. Murray, A. Sangiovanni-Vincentelli, and S. A. Seshia. 2014. "Model predictive control with signal temporal logic specifications". Proc. 53rd IEEE Conference on Decision and Control, pp. 81-87.

Saha, S., and A. A. Julius. 2016. "An MILP approach for real-time optimal controller synthesis with metric temporal logic specifications". Proc. IEEE Amer. Control Conf., pp. 1105-1110.

Sun, H. et al. 2019. "Review of Challenges and Research Opportunities for Voltage Control in Smart Grids". IEEE Trans. Power Syst. vol. 34 (4), pp. 2790-2801.

Wolff, E. M., U. Topcu, and R. M. Murray. 2013. "Automaton-guided controller synthesis for nonlinear systems with temporal logic". Proc. IEEE/RSJ Int. Conf. Intell. Robots Syst, pp. 4332-4339.

Xu, Z., A. Julius, and J. H. Chow. 2019. "Energy Storage Controller Synthesis for Power Systems With Temporal Logic Specifications". IEEE Syst. J. vol. 13 (1), pp. 748-759.

Zhang, Y., M. Olama, A. Melin, Y. Xue, S. Djouadi, and K. Tomsovic. 2018. "Synthesizing distributed energy resources in microgrids with temporal logic specifications". Proc. 9th IEEE International Symposium on Power Electronics for Distributed Generation Systems (PEDG).

Zheng, H., and C. L. DeMarco. 2016. "A New Dynamic Performance Model of Motor Stalling and FIDVR for Smart Grid Monitoring/Planning”. IEEE Trans. Smart Grid vol. 7 (4), pp. 1989-1996.

\section{AUTHOR BIOGRAPHIES}

BYUNGKWON PARK received the B.S. degree in electrical engineering from Chonbuk National University, South Korea, and the Ph.D degree in electrical engineering from the University of Wisconsin-Madison (UW-Madison), in 2011 and 2018, respectively. He is currently a Postdoctoral Research Associate with the Computational Sciences and Engineering Division at Oak Ridge National Laboratory. His research interests include modeling, simulation, control and optimization of smart energy systems. His email address is parkb@ornl.gov.

MOHAMMED M. OLAMA received the B.S. and M.S. (Hons.) degrees in electrical engineering from the University of Jordan, in 1998 and 2001, respectively, and the Ph.D. degree from the Electrical Engineering and Computer Science (EECS) Department, The University of Tennessee, USA, in 2007. He is currently a Research Scientist with the Computational Sciences and Engineering Division, Oak Ridge National Laboratory, USA. He is also an Adjunct Associate Professor with the EECS Department, The University of Tennessee. He has over 100 technical publications. His research interests include smart grid and smart buildings; smart grid communications and control; cyber-physical systems; statistical signal processing; and discrete-event simulation. He received the Best Paper Awards in the IEEE International Symposium on Power Electronics for Distributed Generation Systems, in 2018, and the Mediterranean Conference on Intelligent Systems and Automation, in 2008. His email address is olamahussemm@ornl.gov. 